International Journal of Modern Physics D

(C) World Scientific Publishing Company

\title{
NON-GAUSSIANITY IN THE HILC FOREGROUND-REDUCED THREE-YEAR WMAP CMB MAP
}

\author{
ARMANDO BERNUI* AND MARCELO J. REBOUÇAS \\ Centro Brasileiro de Pesquisas Físicas, Rua Dr. Xavier Sigaud 150 \\ 22290-180 Rio de Janeiro - RJ, Brasil \\ bernui@cbpf.br, reboucas@cbpf.br
}

Received Day Month Year

Revised Day Month Year

Communicated by Managing Editor

\begin{abstract}
A detection or nondetection of primordial non-Gaussianity in the CMB data is essential not only to test alternative models of the physics of the early universe but also to discriminate among classes of inflationary models. Given this far reaching consequences of such a non-Gaussianity detection for our understanding of the physics of the early universe, it is important to employ alternative indicators in order to have further information about the Gaussianity features of CMB that may be helpful for identifying their origins. In this way, a considerable effort has recently gone into the design of non-Gaussianity indicators, and in their application in the search for deviation from Gaussianity in the CMB data. Recently we have proposed two new large-angle non-Gaussianity indicators which provide measures of the departure from Gaussianity on large angular scales. We have used these indicators to carry out analyses of Gaussianity of the single frequency bands and of the available foreground-reduced five-year maps with and without the KQ75 mask. Here we extend and complement these studies by performing a new analysis of deviation from Gaussianity of the three-year harmonic ILC (HILC) foreground-reduced full-sky and KQ75 masked maps obtained from WMAP data. We show that this fullsky foreground-reduced maps presents a significant deviation from Gaussianity, which is brought down to a level of consistency with Gaussianity when the KQ75 mask is employed.
\end{abstract}

Keywords: Gaussianity; cosmic microwave background, inflation, physics of the early universe.

\section{Introduction}

A detection or nondetection of primordial non-Gaussianity in the CMB data is crucial not only to discriminate inflationary models but also to test some alternative scenarios for the physics of the early universe. However, the extraction of primordial non-Gaussianity is a difficult enterprise since several effects of non-primordial nature can produce non-Gaussianity in the CMB data. Clearly the study of detectable non-Gaussianities in the WMAP data must take into account that they

*Permanent address: ICE, Universidade Federal de Itajubá, MG, Brasil 
may have non-cosmological origins as, for example, unsubtracted foreground contamination, unconsidered point sources emission and systematic errors $\frac{112 \mid 3}{}$ Deviation from Gaussianity may also have a cosmic topology origin (see, e.g., the review articles Refs. 4 and related Refs. 5). If, on the one hand, different statistical tools can in principle provide information about distinct forms of non-Gaussianity, on the other hand one does not expect that a single statistical estimator can be sensitive to all possible forms of non-Gaussianity in CMB data. It is therefore important to test CMB data for Gaussianity by using different statistical indicators to shed some light on its possible causes. In view of this, a great deal of effort has recently gone into verifying the existence of non-Gaussianity by employing several statistical estimators. 6

Recently have we proposed 7 two new large-angle non-Gaussianity indicators, based on skewness and kurtosis of large-angle patches of CMB maps, which provide measures of the departure from Gaussianity on large angular scales. We used these indicators to search for the large-angle deviation from Gaussianity in the three and five-year single frequency $\mathrm{K}, \mathrm{Ka}, \mathrm{Q}, \mathrm{V}$, and $\mathrm{W}$ maps with and without a $K Q 75$ mask. We have found strong deviation from Gaussianity in the unmasked maps, whereas a KQ75 mask lowers significantly the level of non-Gaussianity (for details see Ref. (7).

Motivated by the fact that most of Gaussianity analyses with Wilkinson Microwave Anisotropy Probe (WMAP) data have been carried out by using CMB frequency bands masked maps, and that sky cut can in principle induce bias in Gaussianity analyses, in a more recent paper ${ }^{8}$ we have carried out an analysis of Gaussianity of the available full-sky foreground-reduced five-year CMB maps $9 / 10[11$ by using the statistical indicators of Ref. 7 .

We have shown that the available full-sky five-year foreground-reduced maps present a significant deviation from Gaussianity, which varies with the foregroundcleaning procedures. We have also shown that there is a substantial reduction in the level of deviation from Gaussianity in these full sky maps when a $K Q 75$ mask is used. Our main aim here is to extend and complement our previous work ${ }^{8}$ by performing a similar analysis of Gaussianity of the three-year harmonic ILC (HILC) maps 10 foreground-reduced full-sky and KQ75 masked maps. To this end, in the next section we give an account of the large-angle non-Gaussianity indicators of Ref. 7, while in the last section we apply our indicators to perform a Gaussianity analysis of the HILC three-year full-sky and KQ75 cut-sky maps, and present our main results. Our principal conclusion is that the HILC full-sky foreground-reduced maps presents a significant deviation from Gaussianity, which is reduced to a level of consistency with Gaussianity when the KQ75 mask is employed.

\section{Non-Gaussianity Indicators and Associated Maps}

A constructive way of defining our non-Gaussianity indicators $S$ and $K$ (discrete functions defined on $S^{2}$ ) from CMB data can be formalized through the following 
steps

i. Take a finite set of points $\left\{j=1, \ldots, N_{\mathrm{c}}\right\}$ homogeneously distributed on the CMB celestial sphere $S^{2}$ as the centers of spherical caps of a given aperture $\gamma$; and calculate for each cap $j$ the skewness and kurtosis given, respectively, by

$$
S_{j} \equiv \frac{1}{N_{\mathrm{p}} \sigma_{j}^{3}} \sum_{i=1}^{N_{\mathrm{p}}}\left(T_{i}-\overline{T_{j}}\right)^{3} \quad \text { and } \quad K_{j} \equiv \frac{1}{N_{\mathrm{p}} \sigma_{j}^{4}} \sum_{i=1}^{N_{\mathrm{p}}}\left(T_{i}-\overline{T_{j}}\right)^{4}-3,
$$

where $N_{\mathrm{p}}$ is the number of pixels in the $j^{\text {th }}$ cap, $T_{i}$ is the temperature at the $i^{\text {th }}$ pixel, $\overline{T_{j}}$ is the CMB mean temperature of the $j^{\text {th }}$ cap, and $\sigma$ is the standard deviation. Clearly, the numbers $S_{j}$ and $K_{j}$ obtained in this way for each cap can be viewed as a measure of non-Gaussianity in the direction of the center of the $\operatorname{cap}\left(\theta_{j}, \phi_{j}\right)$.

iii. Patching together the $S_{j}$ and $K_{j}$ values for each spherical cap, one obtains our indicators, i.e., discrete functions $S=S(\theta, \phi)$ and $K=K(\theta, \phi)$ defined over the celestial sphere, which can be used to measure the deviation from Gaussianity as a function of the angular coordinates $(\theta, \phi)$. The Mollweide projection of skewness and kurtosis functions $S=S(\theta, \phi)$ and $K=K(\theta, \phi)$ are nothing but skewness and kurtosis maps, hereafter referred to them as $S$-map and $K$-map, respectively.

Clearly, the discrete functions $S=S(\theta, \phi)$ and $K=K(\theta, \phi)$ can be expanded into their spherical harmonics in order to determine their power spectra $S_{\ell}$ and $K_{\ell}$. Thus, for example, for the skewness one has $S(\theta, \phi)=\sum_{\ell=0}^{\infty} \sum_{m=-\ell}^{\ell} b_{\ell m} Y_{\ell m}(\theta, \phi)$ and $S_{\ell}=(2 \ell+1)^{-1} \sum_{m}\left|b_{\ell m}\right|^{2}$. Similar expressions obviously hold for the kurtosis $K=K(\theta, \phi)$.

\section{Main Results and Conclusions}

In this section we shall report the results of our Gaussianity analysis performed with $S=S(\theta, \phi)$ and $K=K(\theta, \phi)$ indicators calculated from the foreground reduced HILC full-sky and KQ75 masked maps computed from three-year WMAP data

To minimize the statistical noise, in the calculations of $S$-map and $K$-map from the HILC foreground-reduced three-year map, we have scanned the celestial sphere with 12288 spherical caps of aperture $\gamma=90^{\circ}$, centered at points homogeneously generated on the two-sphere by using HEALPix 12 .

Figure 1 shows the differential power spectrum of the skewness $S_{\ell}$ (left panel) and kurtosis $K_{\ell}$ (right panel) indicators for $\ell=1, \cdots, 10$, calculated from full-sky

${ }^{\mathrm{a}}$ For a detailed discussion of the indicator briefly presented here we refer the readers to Ref. 7 and Ref. 8 .

${ }^{\mathrm{b}}$ We note that in the analysis of Gaussianity with the $K Q 75$ masked maps the implementation of the mask is made by removing the pixels inside the masked regions from the set of pixels of the each cap whose intersection with the mask is not empty. Thus, the values $S_{j}$ and $K_{j}$ for a $j^{\text {th }}$ cap (with pixels in the mask region) are calculated with small number $N_{\mathrm{p}}$ of pixels. 

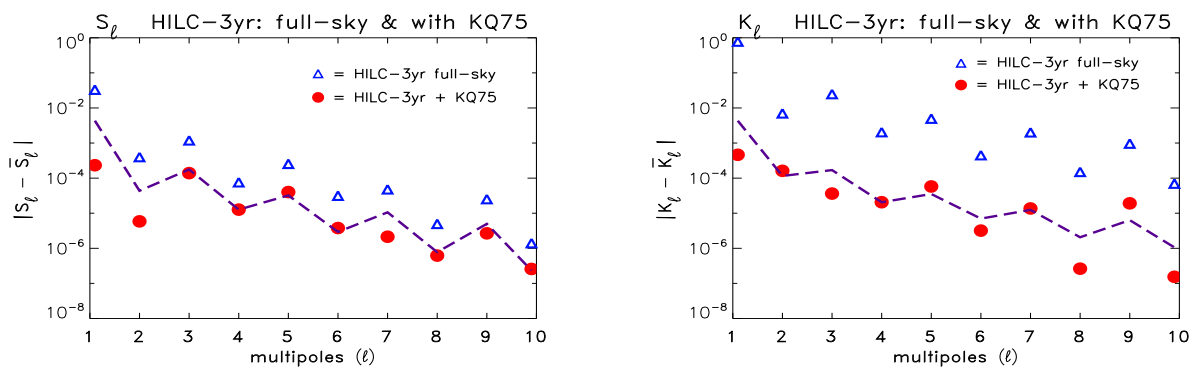

Fig. 1. Low $\ell$ differential power spectra of skewness $\left|S_{\ell}-\bar{S}_{\ell}\right|$ (left) and kurtosis (right) $\mid K_{\ell}-$ $\bar{K}_{\ell} \mid$ calculated from the foreground-reduced HILC full-sky and KQ75 masked maps. The $95 \%$ confidence level (obtained from Monte-Carlo Gaussian maps) is indicated by the dashed line.

and KQ75 cut-sky three-year foreground-reduced HILC maps. The $95 \%$ confidence level, obtained from $S$ and $K$ maps calculated from Monte-Carlo (MC) statistically Gaussian CMB maps, is indicated in this figure $\mathrm{c}$ To the extent that the deviations $\left|S_{\ell}-\bar{S}_{\ell}\right|$ and $\left|K_{\ell}-\bar{K}_{\ell}\right|$ for these maps are not within $95 \%$ of the mean MC value, Fig. 1 shows an important deviation from Gaussianity in full-sky foreground-reduced HILC three-year map. This figure also shows a significant reduction in the level of large-angle deviation from Gaussianity when the $K Q 75$ mask is used.

To have an overall assessment power spectra $S_{\ell}$ and $K_{\ell}$ calculated from the HILC foreground-reduced three-year full and cut map, we have performed a $\chi^{2}$ test to find out the goodness of fit for $S_{\ell}$ and $K_{\ell}$ multipole values as compared to the expected multipole values obtained from $S$ and $K$ maps calculated from Monte-Carlo (MC) statistically Gaussian CMB maps. This gives a number for each case that quantifies collectively the deviation from Gaussianity. For the power spectra $S_{\ell}$ and $K_{\ell}$ we found the values given in Table 1 for the ratio $\chi^{2} /$ dof (dof stands for degrees of freedom) for the power spectra calculated from three-year HILC foreground-reduced full-sky and cut-sky maps.

Table 1. $\quad \chi^{2}$ test goodness of fit for $S_{\ell}$ and $K_{\ell}$ calculated from the HILC full-sky and cut-sky three-year maps as compared with the expected values $\bar{S}_{\ell}$ and $\bar{K}_{\ell}$ obtained from MC maps .

\begin{tabular}{lcc}
\hline Map & $\chi^{2}$ for $S_{\ell}$ & $\chi^{2}$ for $K_{\ell}$ \\
\hline HILC full-sky & $1.6 \times 10^{3}$ & $8.8 \times 10^{5}$ \\
HILC $K Q 75$ cut-sky & 0.8 & 1.5 \\
\hline
\end{tabular}

Clearly, the greater is the values for $\chi^{2} /$ dof the smaller are the $\chi^{2}$ probabilities, that is the probability that the power spectra $S_{\ell}$ and $K_{\ell}$ and the expected MC power spectra agree. Thus, from Table 1 is one concludes that the HILC presents

${ }^{\mathrm{c}}$ For details on the calculation of these (data and MC) maps and the associated power spectra we refer the readers to Ref. $[7$ and Ref. 8 
the substantial level of deviation from Gaussianity as detected by the indicators, which is reduced to a level that can be considered consistent with Gaussianity when the $K Q^{75}$ mask is employed.

Finally we note that the relative deviation of the full-sky power spectrum from the cut-sky spectrum can be calculated with no reference to the Gaussian MC spectra. To this end, we have performed a $\chi^{2}$ test to find out the goodness of fit for $S_{\ell}$ and $K_{\ell}$ multipole values for the full-sky maps as compared to the corresponding cut-sky values. For this relative assessment of power spectra $S_{\ell}$ and $K_{\ell}$ we have found that $\chi^{2} /$ dof are $1.4 \times 10^{3}$ and $7.2 \times 10^{5}$. These values make apparent the significant effect of the mask in the reduction of the deviation from Gaussianity in the full-sky HILC three-year map, and give information on reliability of the HILC full-sky foreground-reduced three-year map as Gaussian reconstruction of the whole CMB sky.

\section{Acknowledgments}

This work is supported by Conselho Nacional de Desenvolvimento Científico e Tecnológico (CNPq) - Brasil, under grant No. 472436/2007-4. M.J.R. and A.B. thank $\mathrm{CNPq}$ for the grants under which this work was carried out. We are also grateful to A.F.F. Teixeira for reading the manuscript and indicating the omissions and misprints. We acknowledge the use of the Legacy Archive for Microwave Background Data Analysis (LAMBDA). Some of the results in this paper were derived using the HEALPix package 12

\section{References}

1. L.-Y. Chiang, P.D. Naselsky, O.V. Verkhodanov, and M.J. Way, Astrophys. J. Suppl 590 (2003) L65.

2. P.D. Naselsky, L.-Y. Chiang, I.D. Novikov, and O.V. Verkhodanov, Int. J. Mod. Phys. D 14 (2005) 1273.

3. P. Cabella, D. Pietrobon, M. Veneziani, A. Balbi, R. Crittenden, G. de Gasperis, C. Quercellini, and N. Vittorio, arXiv:0910.4362

4. G. F. R. Ellis, Gen. Rel. Grav. 2 (1971) 7; M. Lachièze-Rey and J.-P. Luminet, Phys. Rep. 254 (1995) 135; G. D. Starkman, Class. Quantum Grav. 15 (1998) 2529; J.-P. Uzan, R. Lehoucq, and J-P. Luminet, arXiv:gr-qc/0005128, J. Levin, Phys. Rep. 365 (2002) 251; M. J. Rebouças and G. I. Gomero, Braz. J. Phys. 34, 1358 (2004); M. J. Rebouças, arXiv:astro-ph/0504365.

5. G. I. Gomero, M. J. Rebouças and R. Tavakol, Classical Quantum Gravity 18 (2001) 4461: G. I. Gomero, M. J. Rebouças and R. Tavakol, Classical Quantum Gravity 18 (2001) L145; G. I. Gomero, A. F. F. Teixeira, M. J. Rebouças, and A. Bernui, Int. J. Mod. Phys. D 11, 869 (2002); G. I. Gomero, M. J. Rebouças and R. Tavakol, Int. J. Mod. Phys. A 17 (2002) 4261; J. R. Weeks, Mod. Phys. Lett. A 18 (2003) 2099; G. I. Gomero and M. J. Rebouças, Phys. Lett. A 311 (2003) 319; B. Mota, M. J. Rebouças and R. Tavakol, Classical Quantum Gravity 20 (2003) 4837; B. Mota, G. I. Gomero, M. J. Rebouças and R. Tavakol, Classical Quantum Gravity 21 (2004) 3361; B. Mota, M. J. Rebouças and R. Tavakol, Int. J. Mod. Phys. A 20 (2005) 2415; B. 
Mota, M. J. Rebouças and R. Tavakol, Phys. Rev. D 78 (2008) 083521; B. Mota, M. J. Rebouças, and R. Tavakol, arXiv:astro-ph/0403110

6. E. Komatsu et al., Astrophys. J. Suppl 148 (2003) 119; D. N. Spergel et al., Astrophys. J. Suppl. 170 (2007) 377; E. Komatsu et al., Astrophys. J. Suppl. 180 (2009) 330; P. Vielva, E. Martínez-González, R. B. Barreiro, J. L. Sanz and L. Cayón, Astrophys. J. 609 (2004) 22; X. Liu and S. N. Zhang, Astrophys. J. 633 (2005) 542; M. Cruz, E. Martínez-González, P. Vielva and L. Cayón, Mon. Not. R. Astron. Soc. 356 (2005) 29; M. Cruz, L. Cayón, E. Martínez-González, P. Vielva and J. Jin, Astrophys. J. 655 (2007) 11; L. Cayón, J. Jin and A. Treaster, Mon. Not. R. Astron. Soc. 362 (2005) 826; Lung-Y Chiang and P. D. Naselsky, Int. J. Mod. Phys. D 15 (2006) 1283; J. D. McEwen, M. P. Hobson, A N. Lasenby and D. J. Mortlock, Mon. Not. R. Astron. Soc. 371 (2006) L50; J. D. McEwen, M. P. Hobson, A. N. Lasenby and D. J. Mortlock, Mon. Not. R. Astron. Soc. 388 (2008) 659; A. Bernui, C. Tsallis and T. Villela, Europhys. Lett. 78 (2007) 19001; L.-Y. Chiang, P. D. Naselsky and P. Coles, Astrophys. J. 664 (2007) 8; C.-G. Park, Mon. Not. R. Astron. Soc. 349 (2004) 313; H. K. Eriksen, D. I. Novikov, P. B. Lilje, A. J. Banday and K. M. Górski, Astrophys. J. 612 (2004) 64; P. Mukherjee and Y. Wang, Astrophys. J. 613 (2004) 51; M. Cruz, M. Tucci, E. Martínez-González and P. Vielva, Mon. Not. R. Astron. Soc. 369 (2006) 57; A. Bernui, B. Mota, M. J. Rebouças and R. Tavakol, Astron. Astrophys. 464 (2007) 479; M. Cruz, N. Turok, P. Vielva, E. Martínez-González and M. Hobson, Science 318 (2007) 1612; A. Bernui, B. Mota, M. J. Rebouças and R. Tavakol, Int. J. Mod. Phys. D 16 (2007) 411; D. Pietrobon, P. Cabella, A. Balbi, G. de Gasperis and N. Vittorio, Mon. Not. Roy. Astron. Soc. 396 (2009) 1682; D. Pietrobon, P. Cabella, A. Balbi, R. Crittenden, G. de Gasperis and N. Vittorio, arXiv:0905.3702 [astroph.CO]; Y. Ayaita, M. Weber and C. Wetterich, arXiv:0905.3324 [astro-ph.CO]; P. Vielva and J. L. Sanz, Mon. Not. Roy. Astron. Soc. 397 (2009) 837; B. Lew, JCAP 08 (2008) 017; A. Bernui and M. J. Rebouças, Int. J. Mod. Phys. A 24 (2009) 1664; M. Kawasaki, K. Nakayama, T. Sekiguchi, T. Suyama and F. Takahashi, JCAP 11 (2008) 019; M. Kawasaki, K. Nakayama and F. Takahashi, JCAP 01 (2009) 026; M. Kawasaki, K. Nakayama, T. Sekiguchi, T. Suyama and F. Takahashi, JCAP 01 (2009) 042; M. Cruz, E. Martínez-González and P. Vielva, arXiv:0901.1986 [astro-ph.CO].

7. A. Bernui and M. J. Rebouças, Phys. Rev. D 79 (2009) 063528.

8. A. Bernui and M. J. Rebouças, Phys. Rev. D 81 (2010) 063533.

9. G. Hinshaw et al., Astrophys. J. Suppl. Ser. 180 (2009) 225.

10. J. Kim, P. Naselsky and P. R. Christensen, Phys. Rev. D 77 (2008) 103002.

11. J. Delabrouille et al., Astron. Astrophys. 493 (2009) 835.

12. K. M. Górski, E. Hivon, A. J. Banday, B. D. Wandelt, F. K. Hansen, M. Reinecke and M. Bartelman, Astrophys. J. 622 (2005) 759. 